Supporting Information

\title{
On the role played by the chalcogen donor atoms in diimine-dichalcogenolate Pt" SONLO chromophores: is it worth replacing sulfur with selenium?
}

Anna Pintus, M. Carla Aragoni, Francesco Isaia, Vito Lippolis, Dominique Lorcy, Alexandra M. Z. Slawin, J. DerekWoollins, and Massimiliano Arca* 


\section{Experimental part}

Chemicals: 2,2'-Bipyridine, 1,10-phenanthroline, $\mathrm{K}_{2} \mathrm{PtCl}_{4}$, ethyl alcohol, and thf were obtained from commercial sources and purified when necessary by using standard techniques.

Synthesis of Complexes: Platinum dichlorodiimine complexes $\left[\mathrm{Pt}(\right.$ bipy $\left.) \mathrm{Cl}_{2}\right],\left[\mathrm{Pt}(\mathrm{phen}) \mathrm{Cl}_{2}\right],{ }^{1,2}$ and $N$-methyl-4,5-bis(2'-cyanoethylseleno)-1,3-thiazol-2-thione ${ }^{3}$ were synthesized according to published procedures. The complexes $[\mathrm{Pt}($ bipy)(Me-dmet)] (4) and $[\mathrm{Pt}(\mathrm{phen})(\mathrm{Me}-\mathrm{dmet})](\mathbf{5})$ were synthesized and characterized as described in a previous work. ${ }^{4}$

[Pt(bipy)(Me-dset)] (1). A dry ethyl alcohol solution of sodium ethoxyde (15 mL; $3.6 \mathrm{mmol})$ was added dropwise to a solution of $N$-substituted-4,5-bis(2'-cyanoethylseleno)-1,3-thiazol-2-thione in the same solvent (15 mL; $0.18 \mathrm{~g}, 0.45 \mathrm{mmol})$ under a $\mathrm{N}_{2}$ atmosphere. A suspension of [Pt(bipy) $\mathrm{Cl}_{2}$ ] $(0.19 \mathrm{~g}, 0.45 \mathrm{mmol})$ in $20 \mathrm{~mL}$ of dry thf was then added dropwise, and the reaction mixture was left under magnetic stirring for one week, after which the solvent was removed under reduced pressure. The crude product obtained was then suspended in $600 \mathrm{~mL}$ of $\mathrm{CH}_{2} \mathrm{Cl}_{2}$, and $100 \mathrm{~mL}$ of $n$ hexane were added dropwise to the filtered solution, resulting in the precipitation of a dark solid, which was collected by filtration. X-ray quality crystals were obtained by slow infusion of petroleum ether into a $\mathrm{CH}_{2} \mathrm{Cl}_{2}$ solution of the complex. Yield: $0.02 \mathrm{~g}(9 \%)$; m.p. > $240^{\circ} \mathrm{C}$; FT-IR: $\tilde{v}=411(\mathrm{w}), 481(\mathrm{w}), 517(\mathrm{~s}), 638(\mathrm{w}), 712(\mathrm{~s}), 747(\mathrm{vs}), 761(\mathrm{~m}), 789(\mathrm{w}), 906(\mathrm{w}), 925(\mathrm{w}), 990$ (m), 1068 (m), 1124 (vs), 1158 (w), 1246 (w), 1316 (m), 1336 (m), $1384(\mathrm{w}), 1432$ (w), 1446 (s), $1471(\mathrm{~s}), 1498(\mathrm{~m}), 1604(\mathrm{~m}), 3068 \mathrm{~cm}^{-1}$ (vw); UV-Vis-NIR (dmso): $\lambda(\varepsilon)=257$ (17000), 277 (16000), 302 (16000), 331 (6000), 371 (11000), $569 \mathrm{~nm}\left(3500 \mathrm{M}^{-1} \mathrm{~cm}^{-1}\right)$; fluorescence (dmso; $\lambda_{\text {exc }}$ $=341 \mathrm{~nm} ;$ slit $=5 \times 5): \lambda_{e m}=374,394 \mathrm{~nm}, \Phi=8.21 \cdot 10^{-3} ;{ }^{1} \mathrm{H}-\mathrm{NMR}\left(\mathrm{dmso}-d_{6}\right): \delta=9.00(\mathrm{~d}, 1 \mathrm{H})$, $8.90(\mathrm{~d}, 1 \mathrm{H}), 8.79(\mathrm{~d}, 2 \mathrm{H}), 8.54(\mathrm{t}, 2 \mathrm{H}), 8.00(\mathrm{t}, 2 \mathrm{H}), 3.74(\mathrm{~s}, 3 \mathrm{H}) \mathrm{ppm}$; elemental analysis calcd $(\%)$ for $\mathrm{C}_{14} \mathrm{H}_{11} \mathrm{~N}_{3} \mathrm{~S}_{2} \mathrm{Se}_{2} \mathrm{Pt}$ : C 26.34, H 1.74, N 6.58, S 10.05; found: C 25.76, H 1.63, N 6.02, S 9.04; CV (dmso): $E_{1 / 2} v s \mathrm{Fc}^{+} / \mathrm{Fc}\left(\mathrm{scan}\right.$ rate $\left.=50 \mathrm{mV} \mathrm{s}^{-1}\right)=-2.245,-1.607,0.044 \mathrm{~V}$.

[Pt(phen)(Me-dset)] (2). The complex was synthesized as described for [Pt(bipy)(Me-dset)] (1) starting from [Pt(phen)Cl $\mathrm{Cl}_{2}(0.14 \mathrm{~g}, 0.31 \mathrm{mmol})$. Yield: $0.11 \mathrm{~g}(55 \%) ;$ m.p. $>240^{\circ} \mathrm{C}$; FT-IR: $\tilde{v}=$ 
$502(\mathrm{w}), 517(\mathrm{~s}), 650(\mathrm{w}), 705(\mathrm{vs}), 724(\mathrm{~m}), 754(\mathrm{w}), 787(\mathrm{w}), 831(\mathrm{~s}), 916(\mathrm{~m}), 990(\mathrm{~s}), 1063(\mathrm{~m})$, 1126 (s), 1151 (w), 1217 (w), 1294 (w), 1340 (m), 1430 (m), 1502 (m), 1578 cm$^{-1}$ (w); UV-VisNIR (dmso): $\lambda(\varepsilon)=271(41000), 374(16000), 570 \mathrm{~nm}\left(5000 \mathrm{M}^{-1} \mathrm{~cm}^{-1}\right)$; Fluorescence $\left(\mathrm{dmso} ; \lambda_{e c x}\right.$ $=279 \mathrm{~nm} ;$ slit $=5 \times 5): \lambda_{e m}=373,392 \mathrm{~nm}, \Phi=1.18 \cdot 10^{-2} ;{ }^{1} \mathrm{H}$ NMR $\left(\mathrm{dmso}-d_{6}\right): 9.36(\mathrm{~d}, 1 \mathrm{H}), 9.26(\mathrm{~d}$, $1 \mathrm{H}), 9.16(\mathrm{~d}, 2 \mathrm{H}), 8.40(\mathrm{~d}, 2 \mathrm{H}), 8.22(\mathrm{t}, 2 \mathrm{H}), 3.78(\mathrm{~s}, 3 \mathrm{H}) \mathrm{ppm}$; elemental analysis calcd $(\%)$ for $\mathrm{C}_{16} \mathrm{H}_{11} \mathrm{~N}_{3} \mathrm{~S}_{2} \mathrm{Se}_{2} \mathrm{Pt}$ : C 29.01, H 1.67, N 6.34, S 9.68; found: C 30.93, H 2.38, N 7.21, S 8.40; CV $(\mathrm{dmso}): E_{1 / 2} v s \mathrm{Fc}^{+} / \mathrm{Fc}\left(\mathrm{scan}\right.$ rate $\left.=100 \mathrm{mV} \mathrm{s}^{-1}\right)=-1.620,-0.027 \mathrm{~V}$.

Characterization: Elemental analyses were performed with an EA1108 CHNS-O Fisons Instrument $\left(T=1000^{\circ} \mathrm{C}\right)$. FT-IR spectra were recorded with a Thermo-Nicolet 5700 spectrometer at room temperature: $\mathrm{KBr}$ pellets with a $\mathrm{KBr}$ beam-splitter and $\mathrm{KBr}$ windows $\left(4000-400 \mathrm{~cm}^{-1}\right.$, resolution $4 \mathrm{~cm}^{-1}$ ) were used. ${ }^{1} \mathrm{H}-\mathrm{NMR}$ spectra were recorded on a Varian INOVA $400 \mathrm{MHz}$ spectrometer at $298 \mathrm{~K}$ and referenced to $\mathrm{Si}\left(\mathrm{CH}_{3}\right)_{4}$. Absorption spectra were recorded at $298 \mathrm{~K}$ in a quartz cell of $10.00 \mathrm{~mm}$ optical path with a Thermo Evolution 300 (190-1100 nm) spectrophotometer. Uncorrected emission spectra were collected at $298 \mathrm{~K}$ with a Varian Cary Eclipse spectrophotometer equipped with a xenon lamp. Quantum yields were determined relative to anthracene in ethyl alcohol $\left(C=1-5 \cdot 10^{-6} \mathrm{M} ; \lambda_{\mathrm{exc}}=334-359 \mathrm{~nm}\right)$, by calculating the integrated emission intensity of both the sample and the reference through a decomposition of the spectra in their constituent Gaussian curves. Spectral decomposition was carried out by means of the software Fytik. ${ }^{5}$ Cyclic voltammetry measurements (scan rate $10-1000 \mathrm{mV} \mathrm{s}^{-1}$ ) were performed in anhydrous dmso in a Metrohm voltammetric cell, with a combined working and counter platinum electrode and a standard $\mathrm{Ag} / \mathrm{AgCl}$ reference electrode with a Metrohm Autolab PGSTAT 10 potentiostat (supporting electrolyte $\left(\mathrm{TBA}^{+}\right)\left(\mathrm{PF}_{6}^{-}\right) 0.10 \mathrm{M}$ ): reported data are referred to the $\mathrm{Fc}^{+} / \mathrm{Fc}$ reversible couple.

X-ray crystallography: Single crystal X-ray diffraction data for complex $\mathbf{1}$ were collected with the St. Andrews Robotic diffractometer at 125(2) K. 
Insert Ref A. L Fuller, L. A. S. Scott-Hayward, Y. Li, M. Bühl, A. M. Z. Slawin and J. D. Woollins, J. Am. Chem. Soc., 2010, 132 , 5799-5802. DOI: 10.1021/ja100247y

The structures were solved by direct methods with SHELXS $-97^{6}$ and refined on $F^{2}$ by using SHELXL2013.7 I doin't know how to use Endnote.,..the correct Ref for SHELX is now Sheldrick, G. M. (2008). Acta Cryst. A64, 112-122.

DFT Calculations: Theoretical calculations were performed at DFT level ${ }^{8}$ with the Gaussian 09 suite of programs (Rev. A.02) ${ }^{9}$ on a E4 workstation equipped with four quadcore AMD Opteron processors and $16 \mathrm{~Gb}$ of RAM and running the 64 bit version of the Ubuntu 12.04 Linux operating system. The PBE0 (PBE1PBE) hybrid functional was adopted, ${ }^{10}$ and Schäfer, Horn, and Ahlrichs double- $\zeta$ plus polarization all-electron basis sets $^{11}$ were used for $\mathrm{C}, \mathrm{H}, \mathrm{N}, \mathrm{S}$ and $\mathrm{Se}$, whereas CRENBL $\mathrm{BS}^{12}$ with relativistic effective core potentials (RECPs) ${ }^{13,14}$ was chosen for the heavier Pt species. Preliminary calculations were performed on complex 1 by employing the CRENBL-ECP BSs also on the Se atoms, in order to account for the relativistic effects for this atomic species, but no significant improvement was observed to be conferred to the agreement between experimental and calculated data upon employing ECPs,${ }^{15}$ and thus the Ahlrichs BS was eventually employed on all atomic species but the metal. The geometry of the complexes was optimized starting from structural data when available, and tight SCF convergence criterion (SCF=tight keyword) and fine numerical integration grids [Integral(FineGrid) keyword] were used. The nature of the minima of each optimised structure was verified by harmonic frequency calculations (freq=raman keyword). NBO charges distributions ${ }^{16}$ and Wiberg ${ }^{17}$ bond indices were calculated at the optimized geometries. Electronic transition energies and oscillator strength values were calculated at TD-DFT level (100 states). The electronic spectra were simulated by a convolution of Gaussian functions centered at the calculated excitation energies. In order to determine the influence of the solvent on the properties of the 
complexes, solvation calculations were also carried out in dmso, implicitly taken into account by means of the polarizable continuum model (PCM) in its integral equation formalism variant (IEF-PCM). ${ }^{18}$ The total static second-order (quadratic) hyperpolarizability (the first hyperpolarizability) ${ }^{19} \beta_{\text {tot }}$ was calculated as described previously. ${ }^{4,20}$ The programs Gaussview 5.0, ${ }^{21}$ and Molden $5.0^{22}$ were used to investigate the charge distributions and molecular orbital shapes. The software GaussSum $2.1^{23}$ was used to calculate the molecular orbital contributions (MOC) from groups of atoms, along with the contribution of singly excited configurations to each electronic transition, and to generate all the necessary data to simulate absorption spectra. 

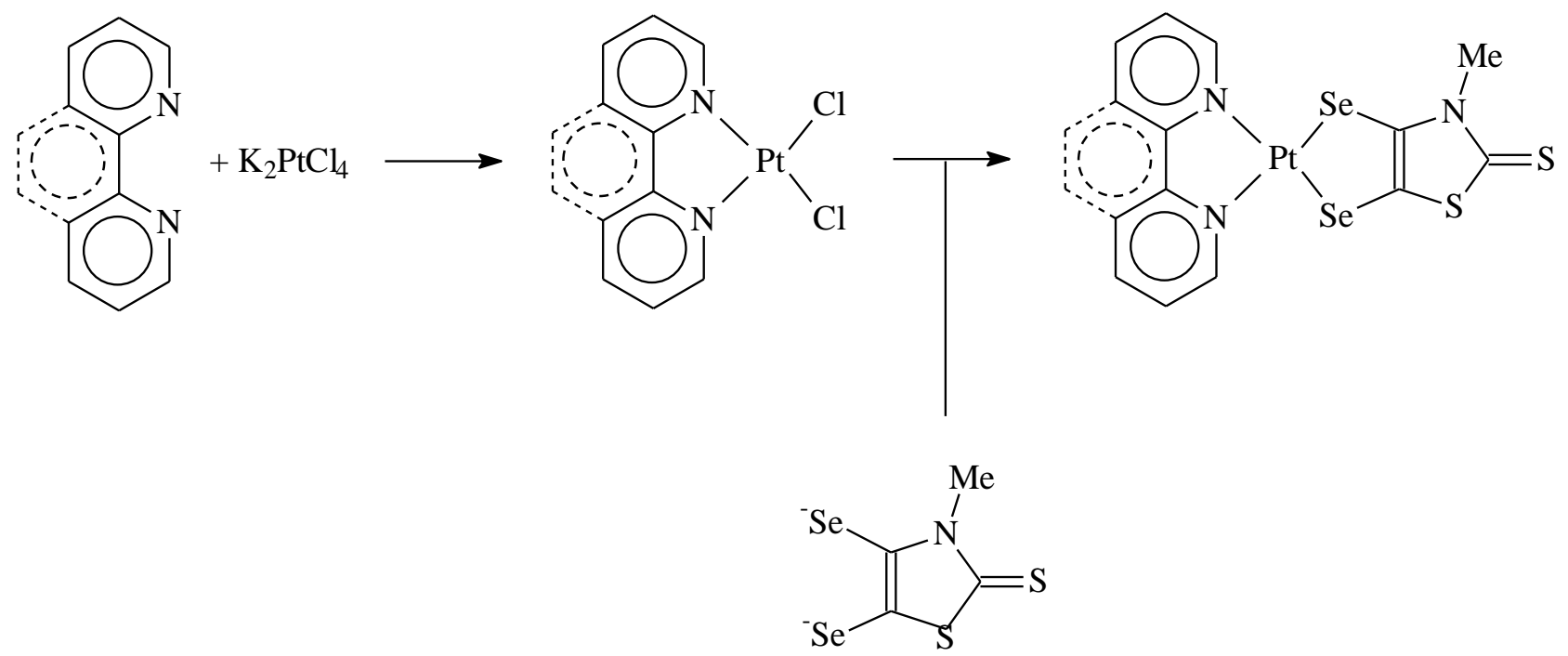

Scheme S1. General pathway for the synthesis of $\left[\mathrm{Pt}\left(\mathrm{N}^{\wedge} \mathrm{N}\right)(\mathrm{Me}-\mathrm{dset})\right]$ complexes 1 and 2. 
Table S1. Crystal data and details of the structure determination for compound $1 \cdot 1 / 2 \mathrm{CH}_{2} \mathrm{Cl}_{2}$.

\begin{tabular}{|c|c|}
\hline Formula & $4.5 \mathrm{H} 12 \mathrm{Cl}$ N3 Pt S2 Se2 \\
\hline Formula Weight & 680.86 \\
\hline Crystal System & Triclinic \\
\hline Space group & (No. 2) \\
\hline $\mathrm{a}, \mathrm{b}, \mathrm{c}[\AA \AA \AA$ & $8.2543(14)$ \\
\hline $105.827(13)$ & $97.865(18)$ \\
\hline$V \quad\left[\AA^{3}\right]$ & $872.2(3)$ \\
\hline $\mathrm{Z}$ & 2 \\
\hline $\mathrm{D}(\mathrm{calc}) \quad\left[\mathrm{g} / \mathrm{cm}^{3}\right]$ & 2.592 \\
\hline $\mathrm{Mu}(\mathrm{MoKa}) \quad[/ \mathrm{mm}]$ & 12.579 \\
\hline$F(000)$ & 630 \\
\hline Crystal Size [mm] & $0.03 \times 0.03 \times 0.15$ \\
\hline Temperature (K) & 125 \\
\hline Radiation $[\AA]$ & 0.71075 \\
\hline Theta Min-Max [ $\left.{ }^{\circ}\right]$ & $2.6, \quad 26.4$ \\
\hline Dataset & $9 ;-10: 10 ;-17: 17$ \\
\hline Tot., Uniq. Data, R(int) & $3441, \quad 0.052$ \\
\hline Observed data $[I>0.0$ sigma (I) ] & 3175 \\
\hline Nref, Npar & $3441, \quad 218$ \\
\hline$R$, $\quad w R 2, \quad S$ & $0.0533,0.1354,1.06$ \\
\hline Max. and Av. Shift/Error & $0.00,0.00$ \\
\hline Min. and Max. Resd. Dens. [e/ $\AA 3$ 3] & $-2.86,2.00$ \\
\hline
\end{tabular}


Table S2. Bond distances $(\AA)$ for compound $1 \cdot 1 / 2 \mathrm{CH}_{2} \mathrm{Cl}_{2}$.

\begin{tabular}{|c|c|c|c|c|c|}
\hline Pt 1 & - Se21 & $2.3929(12)$ & C5 & $-\mathrm{C} 6$ & $1.382(15)$ \\
\hline Pt 1 & $-\mathrm{Se} 22$ & $2.3803(13)$ & $\mathrm{C} 6$ & $-\mathrm{C} 8$ & $1.484(16)$ \\
\hline Pt 1 & $-\mathrm{N} 1$ & $2.078(9)$ & $\mathrm{C} 8$ & $-\mathrm{C} 9$ & $1.391(16)$ \\
\hline Pt1 & $-\mathrm{N} 7$ & $2.060(9)$ & $\mathrm{Cg}$ & $-\mathrm{C} 10$ & $1.407(15)$ \\
\hline $\mathrm{Se} 21$ & $-\mathrm{C} 23$ & $1.883(11)$ & $\mathrm{C} 10$ & $-\mathrm{C} 11$ & $1.393(16)$ \\
\hline Se22 & $-\mathrm{C} 27$ & $1.881(11)$ & $\mathrm{C} 11$ & $-\mathrm{C} 12$ & $1.370(15)$ \\
\hline Cl31 & $-\mathrm{C} 30$ & $1.94(3)$ & $\mathrm{C} 23$ & $-\mathrm{C} 27$ & $1.331(15)$ \\
\hline $\operatorname{Cl} 31$ & $-\mathrm{C} 30 \_\mathrm{a}$ & $1.58(3)$ & $\mathrm{C} 2$ & $-\mathrm{H} 2$ & 0.9500 \\
\hline S24 & $-C 25$ & $1.744(11)$ & $\mathrm{C} 3$ & $-\mathrm{H} 3$ & 0.9500 \\
\hline S24 & $-\mathrm{C} 23$ & $1.740(10)$ & C4 & $-\mathrm{H} 4$ & 0.9500 \\
\hline S27 & $-\mathrm{C} 25$ & $1.662(12)$ & C5 & $-\mathrm{H} 5$ & 0.9500 \\
\hline $\mathrm{N} 1$ & $-\mathrm{C} 6$ & $1.381(14)$ & $\mathrm{C} 9$ & $-\mathrm{H} 9$ & 0.9500 \\
\hline $\mathrm{N} 1$ & $-\mathrm{C} 2$ & $1.323(14)$ & $\mathrm{C} 10$ & $-\mathrm{H} 10$ & 0.9500 \\
\hline N7 & $-\mathrm{C} 8$ & $1.357(14)$ & $\mathrm{C} 11$ & $-\mathrm{H} 11$ & 0.9500 \\
\hline N7 & $-\mathrm{C} 12$ & $1.315(14)$ & $\mathrm{C} 12$ & $-\mathrm{H} 12$ & 0.9500 \\
\hline N2 6 & $-\mathrm{C} 27$ & $1.417(14)$ & $\mathrm{C} 28$ & $-\mathrm{H} 28 \mathrm{C}$ & 0.9800 \\
\hline N26 & $-\mathrm{C} 25$ & $1.369(14)$ & $\mathrm{C} 28$ & $-\mathrm{H} 28 \mathrm{~A}$ & 0.9800 \\
\hline N2 6 & $-\mathrm{C} 28$ & $1.447(14)$ & $\mathrm{C} 28$ & $-\mathrm{H} 28 \mathrm{~B}$ & 0.9800 \\
\hline $\mathrm{C} 2$ & $-\mathrm{C} 3$ & $1.378(16)$ & C30 & $-\mathrm{H} 30 \mathrm{~A}$ & 0.9900 \\
\hline C3 & $-\mathrm{C} 4$ & $1.363(16)$ & C30 & $-\mathrm{H} 30 \mathrm{~B}$ & 0.9900 \\
\hline $\mathrm{C} 4$ & $-\mathrm{C} 5$ & $1.379(16)$ & & & \\
\hline
\end{tabular}

See Table S5 for translation of symmetry code to equivalent positions. 
Table S3. Angles $\left({ }^{\circ}\right.$ ) for compound $1 \cdot 1 / 2 \mathrm{CH}_{2} \mathrm{Cl}_{2}$.

\begin{tabular}{|c|c|c|c|c|c|c|c|}
\hline Se21 & $-P t 1$ & $-\operatorname{Se} 22$ & $91.26(4)$ & N7 & $-\mathrm{C} 8$ & $-\mathrm{C} 6$ & $116.1(10)$ \\
\hline Se21 & - Pt 1 & $-\mathrm{N} 1$ & $174.6(3)$ & $\mathrm{C} 8$ & $-\mathrm{C} 9$ & $-\mathrm{C} 10$ & $119.0(10)$ \\
\hline Se21 & - Pt 1 & $-\mathrm{N} 7$ & $95.0(2)$ & C9 & $-\mathrm{C} 10$ & $-\mathrm{C} 11$ & $117.9(10)$ \\
\hline Se22 & - Pt 1 & $-\mathrm{N} 1$ & $93.3(3)$ & $\mathrm{C} 10$ & $-\mathrm{C} 11$ & $-\mathrm{C} 12$ & $118.7(10)$ \\
\hline Se22 & - Pt 1 & $-\mathrm{N} 7$ & $173.8(2)$ & N7 & $-\mathrm{C} 12$ & $-\mathrm{C} 11$ & $124.4(10)$ \\
\hline $\mathrm{N} 1$ & $-P t 1$ & $-\mathrm{N} 7$ & $80.5(4)$ & $\operatorname{se} 21$ & $-\mathrm{C} 23$ & $-\mathrm{C} 27$ & $123.0(8)$ \\
\hline Pt 1 & $-\operatorname{Se} 21$ & $-\mathrm{C} 23$ & $100.2(3)$ & S24 & $-\mathrm{C} 23$ & $-\mathrm{C} 27$ & $110.1(8)$ \\
\hline Pt1 & -Se22 & $-\mathrm{C} 27$ & $100.1(3)$ & $\operatorname{Se} 21$ & $-C 23$ & $-S 24$ & $126.9(6)$ \\
\hline C30 & $-\mathrm{Cl} 31$ & $-\mathrm{C} 30 \_\mathrm{a}$ & $68.2(14)$ & S24 & $-\mathrm{C} 25$ & $-\mathrm{N} 26$ & $108.4(8)$ \\
\hline $\mathrm{C} 23$ & $-S 24$ & $-\mathrm{C} 25^{-}$ & $93.0(5)$ & S27 & $-\mathrm{C} 25$ & $-\mathrm{N} 26$ & $127.7(8)$ \\
\hline Pt1 & $-\mathrm{N} 1$ & $-\mathrm{C} 2$ & $127.0(8)$ & S24 & $-C 25$ & $-S 27$ & $123.9(7)$ \\
\hline C2 & $-\mathrm{N} 1$ & $-\mathrm{C} 6$ & $119.7(9)$ & $\mathrm{Se} 22$ & $-\mathrm{C} 27$ & $-\mathrm{C} 23$ & $124.1(8)$ \\
\hline Pt1 & $-\mathrm{N} 1$ & $-\mathrm{C} 6$ & $113.2(7)$ & N2 6 & $-\mathrm{C} 27$ & $-\mathrm{C} 23$ & $114.2(9)$ \\
\hline Pt 1 & $-\mathrm{N} 7$ & $-\mathrm{C} 8$ & $114.4(7)$ & $\mathrm{Se} 22$ & $-\mathrm{C} 27$ & $-\mathrm{N} 26$ & $121.6(7)$ \\
\hline Pt 1 & $-\mathrm{N} 7$ & $-\mathrm{C} 12$ & $127.1(7)$ & C3 & $-\mathrm{C} 2$ & $-\mathrm{H} 2$ & 119.00 \\
\hline C8 & $-\mathrm{N} 7$ & $-\mathrm{C} 12$ & $118.4(10)$ & N1 & $-\mathrm{C} 2$ & $-\mathrm{H} 2$ & 119.00 \\
\hline C25 & $-\mathrm{N} 26$ & $-\mathrm{C} 27$ & $114.3(8)$ & $\mathrm{C} 2$ & $-\mathrm{C} 3$ & $-\mathrm{H} 3$ & 121.00 \\
\hline C25 & $-\mathrm{N} 26$ & $-\mathrm{C} 28$ & $122.2(9)$ & $\mathrm{C} 4$ & $-\mathrm{C} 3$ & $-\mathrm{H} 3$ & 121.00 \\
\hline C27 & $-\mathrm{N} 26$ & $-\mathrm{C} 28$ & $123.5(9)$ & $\mathrm{C} 5$ & $-\mathrm{C} 4$ & $-\mathrm{H} 4$ & 120.00 \\
\hline N1 & $-\mathrm{C} 2$ & $-\mathrm{C} 3$ & $122.2(11)$ & $\mathrm{C} 3$ & $-\mathrm{C} 4$ & $-\mathrm{H} 4$ & 120.00 \\
\hline $\mathrm{C} 2$ & $-\mathrm{C} 3$ & $-\mathrm{C} 4$ & $118.7(11)$ & $\mathrm{C} 4$ & $-\mathrm{C} 5$ & $-\mathrm{H} 5$ & 120.00 \\
\hline $\mathrm{C} 3$ & $-\mathrm{C} 4$ & $-\mathrm{C} 5$ & $120.5(10)$ & $\mathrm{C} 6$ & $-\mathrm{C} 5$ & $-\mathrm{H} 5$ & 120.00 \\
\hline $\mathrm{C} 4$ & $-\mathrm{C} 5$ & $-\mathrm{C} 6$ & $119.0(11)$ & $\mathrm{C} 10$ & $-\mathrm{C} 9$ & $-\mathrm{H} 9$ & 120.00 \\
\hline $\mathrm{N} 1$ & $-\mathrm{C} 6$ & $-\mathrm{C} 8$ & $115.4(9)$ & $\mathrm{C} 8$ & $-\mathrm{C} 9$ & $-\mathrm{H} 9$ & 121.00 \\
\hline $\mathrm{C} 5$ & $-\mathrm{C} 6$ & $-\mathrm{C} 8$ & $124.8(10)$ & $\mathrm{Cg}$ & $-\mathrm{C} 10$ & $-\mathrm{H} 10$ & 121.00 \\
\hline $\mathrm{N} 1$ & $-\mathrm{C} 6$ & $-\mathrm{C} 5$ & $119.8(10)$ & $\mathrm{C} 11$ & $-\mathrm{C} 10$ & $-\mathrm{H} 10$ & 121.00 \\
\hline N7 & $-\mathrm{C} 8$ & $-\mathrm{C9}$ & $121.6(10)$ & $\mathrm{C} 12$ & $-\mathrm{C} 11$ & $-\mathrm{H} 11$ & 121.00 \\
\hline $\mathrm{C} 6$ & $-\mathrm{C} 8$ & $-\mathrm{C} 9$ & $122.4(10)$ & $\mathrm{C} 10$ & $-\mathrm{C} 11$ & $-\mathrm{H} 11$ & 121.00 \\
\hline N7 & $-\mathrm{C} 12$ & $-\mathrm{H} 12$ & 118.00 & $\mathrm{H} 28 \mathrm{~A}$ & $-\mathrm{C} 28$ & $-\mathrm{H} 28 \mathrm{~B}$ & 109.00 \\
\hline C11 & $-\mathrm{C} 12$ & $-\mathrm{H} 12$ & 118.00 & C131 & $-C 30$ & $-\operatorname{cl31} a$ & $111.8(16)$ \\
\hline N26 & $-\mathrm{C} 28$ & $-\mathrm{H} 28 \mathrm{~B}$ & 109.00 & Cl31 & $-\mathrm{C} 30$ & $-\mathrm{H} 30 \mathrm{~A}^{-}$ & 109.00 \\
\hline N26 & $-\mathrm{C} 28$ & $-\mathrm{H} 28 \mathrm{C}$ & 109.00 & Cl31 & $-C 30$ & $-\mathrm{H} 30 \mathrm{~B}$ & 109.00 \\
\hline N2 6 & $-\mathrm{C} 28$ & $-\mathrm{H} 28 \mathrm{~A}$ & 109.00 & $\mathrm{H} 30 \mathrm{~A}$ & $-\mathrm{C} 30$ & $-\mathrm{H} 30 \mathrm{~B}$ & 108.00 \\
\hline $\mathrm{H} 28 \mathrm{~A}$ & $-\mathrm{C} 28$ & $-\mathrm{H} 28 \mathrm{C}$ & 109.00 & Cl31_a & $-\mathrm{C} 30$ & $-\mathrm{H} 30 \mathrm{~A}$ & 109.00 \\
\hline $\mathrm{H} 28 \mathrm{~B}$ & $-\mathrm{C} 28$ & $-\mathrm{H} 28 \mathrm{C}$ & 109.00 & Cl31_a & $-\mathrm{C} 30$ & $-\mathrm{H} 30 \mathrm{~B}$ & 109.00 \\
\hline
\end{tabular}

See Table S5 for translation of symmetry code to equivalent positions. 
Table S4. Torsion angles $\left(^{\circ}\right)$ for compound $1 \cdot 1 / 2 \mathrm{CH}_{2} \mathrm{Cl}_{2}$.

\begin{tabular}{|c|c|c|c|c|}
\hline Se22 & -Pt 1 & $-\mathrm{Se} 21$ & $-C 23$ & $9.8(3)$ \\
\hline N7 & -Pt 1 & $-\mathrm{Se} 21$ & $-\mathrm{C} 23$ & $-169.6(4)$ \\
\hline Se21 & $-\mathrm{Pt} 1$ & $-\mathrm{Se} 22$ & $-\mathrm{C} 27$ & $-9.6(3)$ \\
\hline N1 & -Pt 1 & $-\mathrm{Se} 22$ & $-\mathrm{C} 27$ & $173.2(4)$ \\
\hline Se22 & - Pt 1 & $-\mathrm{N} 1$ & $-\mathrm{C} 2$ & $-1.6(9)$ \\
\hline Se22 & -Pt1 & $-\mathrm{N} 1$ & $-\mathrm{C} 6$ & $175.0(7)$ \\
\hline N7 & -Pt1 & $-\mathrm{N} 1$ & $-\mathrm{C} 2$ & $177.5(10)$ \\
\hline N7 & -Pt1 & $-\mathrm{N} 1$ & $-\mathrm{C} 6$ & $-5.9(7)$ \\
\hline Se21 & -Pt 1 & $-\mathrm{N} 7$ & $-\mathrm{C} 8$ & $-172.4(7)$ \\
\hline Se21 & - Pt 1 & $-\mathrm{N} 7$ & $-\mathrm{C} 12$ & $5.2(9)$ \\
\hline N1 & -Pt 1 & $-\mathrm{N} 7$ & $-\mathrm{C} 8$ & $4.7(7)$ \\
\hline N1 & - Pt 1 & $-\mathrm{N} 7$ & $-\mathrm{C} 12$ & $-177.7(9)$ \\
\hline Pt1 & $-\mathrm{Se} 21$ & $-\mathrm{C} 23$ & $-S 24$ & $172.0(6)$ \\
\hline Pt1 & $-\mathrm{Se} 21$ & $-\mathrm{C} 23$ & $-\mathrm{C} 27$ & $-8.4(9)$ \\
\hline Pt1 & $-\mathrm{Se} 22$ & $-\mathrm{C} 27$ & $-\mathrm{N} 26$ & $-175.1(8)$ \\
\hline Pt 1 & $-\operatorname{Se} 22$ & $-\mathrm{C} 27$ & $-\mathrm{C} 23$ & $7.9(10)$ \\
\hline C30_a & $-\mathrm{Cl} 31$ & $-\mathrm{C} 30$ & -Cl31_a & $0.0(15)$ \\
\hline $\mathrm{C} 30^{-}$ & $-\operatorname{Cl} 31$ & $-\mathrm{C} 30 \_a$ & $-\operatorname{cl31} 1^{-} \mathrm{a}$ & $0.0(13)$ \\
\hline C25 & $-\mathrm{S} 24$ & $-\mathrm{C} 23^{-}$ & $-\mathrm{Se} 21^{-}$ & $178.0(7)$ \\
\hline C23 & $-\mathrm{S} 24$ & $-\mathrm{C} 25$ & $-\mathrm{N} 26$ & $0.0(8)$ \\
\hline C25 & $-S 24$ & $-\mathrm{C} 23$ & $-\mathrm{C} 27$ & $-1.7(9)$ \\
\hline C23 & $-\mathrm{S} 24$ & $-\mathrm{C} 25$ & $-S 27$ & $179.1(8)$ \\
\hline Pt1 & $-\mathrm{N} 1$ & $-\mathrm{C} 6$ & $-\mathrm{C} 5$ & $-173.3(8)$ \\
\hline Pt1 & $-\mathrm{N} 1$ & $-\mathrm{C} 6$ & $-\mathrm{C} 8$ & $6.2(11)$ \\
\hline C2 & $-\mathrm{N} 1$ & $-\mathrm{C} 6$ & $-\mathrm{C} 5$ & $3.6(15)$ \\
\hline C2 & $-\mathrm{N} 1$ & $-\mathrm{C} 6$ & $-\mathrm{C} 8$ & $-176.9(10)$ \\
\hline Pt1 & $-\mathrm{N} 1$ & $-\mathrm{C} 2$ & $-\mathrm{C} 3$ & $173.3(8)$ \\
\hline $\mathrm{C} 6$ & $-\mathrm{N} 1$ & $-\mathrm{C} 2$ & $-\mathrm{C} 3$ & $-3.1(17)$ \\
\hline Pt1 & $-\mathrm{N} 7$ & $-\mathrm{C} 8$ & $-\mathrm{C} 6$ & $-2.8(11)$ \\
\hline C12 & $-\mathrm{N} 7$ & $-\mathrm{C} 8$ & $-\mathrm{C} 9$ & $0.7(15)$ \\
\hline Pt1 & $-\mathrm{N} 7$ & $-\mathrm{C} 12$ & $-\mathrm{C} 11$ & $-177.4(8)$ \\
\hline C8 & $-\mathrm{N} 7$ & $-\mathrm{C} 12$ & $-\mathrm{C} 11$ & $0.1(16)$ \\
\hline Pt1 & $-\mathrm{N} 7$ & $-\mathrm{C} 8$ & $-\mathrm{C} 9$ & $178.6(8)$ \\
\hline C12 & $-\mathrm{N} 7$ & $-\mathrm{C} 8$ & $-\mathrm{C} 6$ & $179.4(9)$ \\
\hline C27 & $-\mathrm{N} 26$ & $-\mathrm{C} 25$ & $-S 24$ & $1.6(11)$ \\
\hline C28 & $-\mathrm{N} 26$ & $-\mathrm{C} 25$ & $-S 27$ & $1.6(16)$ \\
\hline C27 & $-\mathrm{N} 26$ & $-\mathrm{C} 25$ & $-S 27$ & $-177.5(9)$ \\
\hline C28 & $-\mathrm{N} 26$ & $-\mathrm{C} 25$ & - S24 & $-179.4(9)$ \\
\hline C28 & $-\mathrm{N} 26$ & $-\mathrm{C} 27$ & -Se22 & $0.7(14)$ \\
\hline C28 & $-\mathrm{N} 26$ & $-\mathrm{C} 27$ & $-\mathrm{C} 23$ & $177.9(10)$ \\
\hline C25 & $-\mathrm{N} 26$ & $-\mathrm{C} 27$ & -Se22 & $179.7(8)$ \\
\hline C25 & $-\mathrm{N} 26$ & $-\mathrm{C} 27$ & $-\mathrm{C} 23$ & $-3.0(13)$ \\
\hline N1 & $-\mathrm{C} 2$ & $-\mathrm{C} 3$ & $-\mathrm{C} 4$ & $1.9(18)$ \\
\hline $\mathrm{C} 2$ & $-\mathrm{C} 3$ & $-\mathrm{C} 4$ & $-\mathrm{C} 5$ & $-1.2(17)$ \\
\hline C3 & $-\mathrm{C} 4$ & $-\mathrm{C} 5$ & $-\mathrm{C} 6$ & $1.7(17)$ \\
\hline $\mathrm{C} 4$ & $-\mathrm{C} 5$ & $-\mathrm{C} 6$ & $-\mathrm{C} 8$ & $177.7(10)$ \\
\hline $\mathrm{C} 4$ & $-\mathrm{C} 5$ & $-\mathrm{C} 6$ & $-\mathrm{N} 1$ & $-2.8(16)$ \\
\hline N1 & $-\mathrm{C} 6$ & $-\mathrm{C} 8$ & $-\mathrm{C} 9$ & $176.3(10)$ \\
\hline C5 & $-\mathrm{C} 6$ & $-\mathrm{C} 8$ & $-\mathrm{N} 7$ & $177.2(10)$ \\
\hline N1 & $-\mathrm{C} 6$ & $-\mathrm{C} 8$ & $-\mathrm{N} 7$ & $-2.3(14)$ \\
\hline C5 & $-\mathrm{C} 6$ & $-\mathrm{C} 8$ & $-\mathrm{C} 9$ & $-4.2(17)$ \\
\hline C6 & $-\mathrm{C} 8$ & $-\mathrm{C} 9$ & $-\mathrm{C} 10$ & $179.9(10)$ \\
\hline N7 & $-\mathrm{C} 8$ & $-\mathrm{C} 9$ & $-\mathrm{C} 10$ & $-1.6(16)$ \\
\hline C8 & $-\mathrm{C} 9$ & $-\mathrm{C} 10$ & $-\mathrm{C} 11$ & $1.6(16)$ \\
\hline C9 & $-\mathrm{C} 10$ & $-\mathrm{C} 11$ & $-\mathrm{C} 12$ & $-0.9(16)$ \\
\hline C10 & $-\mathrm{C} 11$ & $-\mathrm{C} 12$ & $-\mathrm{N} 7$ & $0.0(17)$ \\
\hline Se21 & $-\mathrm{C} 23$ & $-\mathrm{C} 27$ & -Se22 & $0.4(14)$ \\
\hline S24 & $-\mathrm{C} 23$ & $-\mathrm{C} 27$ & $-\mathrm{N} 26$ & $2.9(12)$ \\
\hline Se21 & $-\mathrm{C} 23$ & $-\mathrm{C} 27$ & $-\mathrm{N} 26$ & $-176.8(7)$ \\
\hline S24 & $-\mathrm{C} 23$ & $-\mathrm{C} 27$ & $-\operatorname{se} 22$ & $-179.9(6)$ \\
\hline
\end{tabular}

See Table S5 for translation of symmetry code to equivalent positions. 
Table S5. Translation of symmetry code to equivalent positions for compound $1 \cdot 1 / 2 \mathrm{CH}_{2} \mathrm{Cl}_{2}$.
$a=[2666.00]=\left[2 \_666\right]=1-x, 1-y, 1-z$
$\mathrm{b}=[2667.00]=\left[2 \_667\right]=1-x, 1-y, 2-z$
$C=[2567.00]=\left[2 \_567\right]=-x, 1-y, 2-z$
$\mathrm{d}=\left[\begin{array}{ll}1565.00\end{array}\right]=\left[1{ }_{-} 565\right]=\mathrm{x}, 1+\mathrm{y}, \mathrm{z}$
$e=[2577.00]=\left[2 \_577\right]=-x, 2-y, 2-z$
$f=[2566.00]=\left[2 \_566\right]=-x, 1-y, 1-z$
$g=[2576.00]=\left[2 \_576\right]=-x, 2-y, 1-z$
$\mathrm{h}=[2576.00]=\left[2 \_576\right]=-\mathrm{x}, 2-\mathrm{y}, 1-\mathrm{z}$
$j=[1545.00]=\left[1 \_545\right]=x,-1+y, z$
$k=[2657.00]=\left[2 \_657\right]=1-x,-y, 2-z$
$I=[1556.00]=\left[1 \_556\right]=\mathrm{X}, \mathrm{y}, 1+\mathrm{z}$
$\mathrm{m}=[1554.00]=\left[1 \_554\right]=\mathrm{x}, \mathrm{y},-1+\mathrm{z}$
$\mathrm{n}=[2666.00]=\left[2 \_666\right]=1-\mathrm{x}, 1-\mathrm{y}, 1-\mathrm{z}$ 
Table S6. Half-wave potentials $\left(\mathrm{V} v s \mathrm{Fc}^{+} / \mathrm{Fc}\right)$ and electrochemical data recorded by $\mathrm{CV}$ on dmso solutions of complexes 1 and $\mathbf{2}$ (scan rate $50 \mathrm{mV} \mathrm{s}^{-1}$ ).

\begin{tabular}{cccc}
\hline & & $\mathbf{1}$ & $\mathbf{2}$ \\
& $E_{1 / 2}$ & 0.044 & -0.027 \\
$E_{1 / 2}{ }^{I}$ & $\left|E_{p c}-E_{p a}\right|$ & 0.054 & 0.052 \\
& $i_{p c} / i_{p a}$ & 0.5 & 0.9 \\
& $E_{1 / 2}$ & -1.607 & -1.620 \\
$E_{1 / 2}{ }^{I I}$ & $\left|E_{p c}-E_{p a}\right|$ & 0.049 & 0.056 \\
& $i_{p c} / i_{p a}$ & 1.2 & 1.1 \\
& $E_{1 / 2}$ & -2.245 & $/$ \\
$E_{1 / 2}{ }^{I I I}$ & $\left|E_{p c}-E_{p a}\right|$ & 0.048 & $/$ \\
& $i_{p c} / i_{p a}$ & 0.8 & $/$ \\
\hline
\end{tabular}


Table S7. Selected optimized bond lengths $(\AA)$ and angles $\left(^{\circ}\right)$ for complexes $\mathbf{1}$ and $\mathbf{2}$ in the gas phase and in $\mathrm{CH}_{2} \mathrm{Cl}_{2}$ (IEF-PCM SCRF model, in parenthesis). ${ }^{\mathrm{a}}$

\begin{tabular}{lcc}
\hline & $\mathbf{1}$ & $\mathbf{2}$ \\
& & \\
\hline $\mathrm{Pt}(13)-\mathrm{Se}(22)$ & 2.387 & 2.385 \\
& $(2.401)$ & $(2.398)$ \\
$\mathrm{Pt}(13)-\mathrm{Se}(23)$ & 2.385 & 2.382 \\
& $(2.401)$ & $(2.398)$ \\
$\operatorname{Pt}(13)-\mathrm{N}(1)$ & 2.059 & 2.066 \\
& $(2.068)$ & $(2.075)$ \\
$\operatorname{Pt}(13)-\mathrm{N}(7)$ & 2.058 & 2.064 \\
& $(2.067)$ & $(2.072)$ \\
$\mathrm{Se}(22)-\mathrm{C}(27)$ & 1.880 & 1.881 \\
& $(1.87)$ & $(1.888)$ \\
$\mathrm{Se}(21)-\mathrm{C}(23)$ & 1.871 & 1.872 \\
& $(1.879)$ & $(1.880)$ \\
$\mathrm{C}(27)-\mathrm{C}(23)$ & 1.359 & 1.359 \\
& $(1.358)$ & $(1.358)$ \\
$\mathrm{Se}(22)-\mathrm{Pt}(13)-\operatorname{Se}(21)$ & 90.71 & 91.12 \\
& $(90.97)$ & $(91.38)$ \\
$\mathrm{N}(1)-\mathrm{Pt}(13)-\mathrm{N}(7)$ & 79.20 & 79.97 \\
& $(79.21)$ & $(79.99)$ \\
$\mathrm{N}(1)-\mathrm{Pt}-\mathrm{Se}(21)-\mathrm{C}(23)$ & 0.00 & 0.00 \\
& $(0.00)$ & $(0.00)$ \\
\hline
\end{tabular}

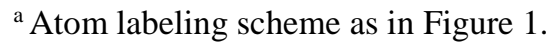


Table S8. Calculated eigenvalues $E(\mathrm{eV})$ and composition (\%) of frontier KS-MOs in terms of the central Pt atom and the $\mathrm{N}^{\wedge} \mathrm{N}$ and Me-dset ${ }^{2-}$ ligands, and HOMO-LUMO energy gaps (eV) for complexes $\mathbf{1}$ and $\mathbf{2}$ in the gas phase and in $\mathrm{CH}_{2} \mathrm{Cl}_{2}$ (IEF-PCM SCRF model).

\begin{tabular}{|c|c|c|c|c|c|c|c|}
\hline & & $\triangle E_{\text {номо-цUмо }}$ & & $E$ & $\mathrm{~N}^{\wedge} \mathrm{N}$ & ${\mathrm{Me}-\mathrm{dset}^{2-}}^{2-}$ & $\mathrm{Pt}$ \\
\hline \multirow[b]{2}{*}{ Gas } & 1 & 1.55 & $\begin{array}{l}\text { HOMO } \\
\text { LUMO }\end{array}$ & $\begin{array}{l}-4.57 \\
-3.02\end{array}$ & $\begin{array}{l}10 \\
85\end{array}$ & $\begin{array}{l}88 \\
9\end{array}$ & $\begin{array}{l}3 \\
6\end{array}$ \\
\hline & 2 & 2.31 & $\begin{array}{l}\text { HOMO } \\
\text { LUMO }\end{array}$ & $\begin{array}{l}-4.53 \\
-2.97\end{array}$ & $\begin{array}{l}9 \\
86\end{array}$ & $\begin{array}{l}88 \\
9\end{array}$ & $\begin{array}{l}3 \\
6\end{array}$ \\
\hline \multirow{2}{*}{$\mathrm{CH}_{2} \mathrm{Cl}_{2}$} & 1 & 1.56 & $\begin{array}{l}\text { HOMO } \\
\text { LUMO }\end{array}$ & $\begin{array}{l}-5.00 \\
-2.69\end{array}$ & $\begin{array}{l}4 \\
93\end{array}$ & $\begin{array}{l}91 \\
3\end{array}$ & $\begin{array}{l}5 \\
4\end{array}$ \\
\hline & 2 & 2.34 & $\begin{array}{l}\text { HOMO } \\
\text { LUMO }\end{array}$ & $\begin{array}{l}-4.99 \\
-2.65\end{array}$ & $\begin{array}{l}4 \\
93\end{array}$ & $\begin{array}{l}91 \\
3\end{array}$ & $\begin{array}{l}5 \\
4\end{array}$ \\
\hline
\end{tabular}


Table S9. NPA charges $Q$ (e) calculated on Pt, Se(21), Se(22), N(1), N(7), the Me-dset ${ }^{2-}$ ligand and the diimmine $\mathrm{N}^{\wedge} \mathrm{N}$ for $\mathbf{1}$ and $\mathbf{2}$ in the gas phase and in $\mathrm{CH}_{2} \mathrm{Cl}_{2}$ (IEF-PCM SCRF model). ${ }^{\mathrm{a}}$

\begin{tabular}{|c|c|c|c|}
\hline & & 1 & 2 \\
\hline \multirow{7}{*}{ Gas } & $Q(\mathrm{Pt} 13)$ & 0.051 & 0.049 \\
\hline & $Q(\mathrm{~N} 1)$ & -0.452 & -0.448 \\
\hline & $Q(\mathrm{~N} 7)$ & -0.454 & -0.451 \\
\hline & $Q(\mathrm{Se} 22)$ & 0.074 & 0.074 \\
\hline & $Q(\mathrm{Se} 21)$ & 0.133 & 0.132 \\
\hline & $Q\left(\mathrm{~N}^{\wedge} \mathrm{N}\right)$ & 0.305 & 0.314 \\
\hline & $Q($ Me-dset $)$ & -0.357 & -0.363 \\
\hline \multirow{7}{*}{$\mathrm{CH}_{2} \mathrm{Cl}_{2}$} & $Q(\mathrm{Pt} 13)$ & 0.061 & 0.059 \\
\hline & $Q(\mathrm{~N} 1)$ & -0.451 & -0.447 \\
\hline & $Q(\mathrm{~N} 7)$ & -0.450 & -0.448 \\
\hline & $Q(\mathrm{Se} 22)$ & 0.057 & 0.022 \\
\hline & $Q(\operatorname{Se} 21)$ & 0.019 & 0.060 \\
\hline & $Q\left(\mathrm{~N}^{\wedge} \mathrm{N}\right)$ & 0.506 & 0.505 \\
\hline & $Q($ Me-dset $)$ & -0.567 & -0.564 \\
\hline
\end{tabular}

${ }^{\text {a }}$ Atom labelling scheme as in Figure 1. 
Table S10. Main electronic transitions $(f>0.010)$ calculated for 1 in $\mathrm{CH}_{2} \mathrm{Cl}_{2}$ (IEF-PCM SCRF model) at the TD-DFT level. For each transition, the excitation energy $E(\mathrm{eV})$, the absorption wavelength $\lambda(\mathrm{nm})$, the oscillator strength $f$, and the molecular orbital composition of the excitestate functions, along with the fragments where the involved KS-MOs are mainly localized, are reported.

\begin{tabular}{|c|c|c|c|c|c|c|}
\hline Exc. state & $E$ & $\lambda$ & $f$ & Composition $^{\mathrm{a}}$ & $\%$ & Assignment \\
\hline S1 & 1.751 & 708.0 & 0.135 & $117 \rightarrow 118$ & 99 & $\operatorname{Me}^{2} \operatorname{dset}^{2-}(91 \%) \rightarrow \operatorname{bipy}(93 \%)$ \\
\hline S5 & 2.849 & 435.2 & 0.012 & $117 \rightarrow 120$ & 97 & Me-dset $^{2-}(91 \%) \rightarrow \operatorname{bipy}(98 \%)$ \\
\hline S8 & 3.149 & 393.7 & 0.037 & $114 \rightarrow 118$ & 96 & $\operatorname{Me}^{-\operatorname{dset}^{2-}(82 \%) \rightarrow \operatorname{bipy}(93 \%)}$ \\
\hline S11 & 3.691 & 335.9 & 0.227 & $117 \rightarrow 122$ & 83 & Me-dset $^{2-}(91 \%) \rightarrow$ Me-dset $^{2-}(99 \%)$ \\
\hline \multirow[t]{2}{*}{$\mathrm{S} 12$} & 3.790 & 327.1 & 0.067 & $113 \rightarrow 121$ & 62 & $\operatorname{Pt}(93 \%) \rightarrow$ Me-dset $^{2-}(42 \%)+\operatorname{Pt}(39 \%)$ \\
\hline & & & & $116 \rightarrow 120$ & 30 & Me-dset $^{2-}(75 \%) \rightarrow \operatorname{bipy}(98 \%)$ \\
\hline \multirow[t]{2}{*}{ S13 } & 3.800 & 326.3 & 0.012 & $116 \rightarrow 120$ & 61 & Me-dset $^{2-}(75 \%) \rightarrow \operatorname{bipy}(98 \%)$ \\
\hline & & & & $113 \rightarrow 121$ & 22 & $\operatorname{Pt}(93 \%) \rightarrow$ Me-dset $^{2-}(42 \%)+\operatorname{Pt}(39 \%)$ \\
\hline S17 & 4.146 & 299.0 & 0.017 & $114 \rightarrow 119$ & 95 & $\operatorname{Me} \operatorname{dset}^{2-}(82 \%) \rightarrow \operatorname{bipy}(98 \%)$ \\
\hline \multirow[t]{2}{*}{ S19 } & 4.258 & 291.1 & 0.043 & $114 \rightarrow 120$ & 52 & 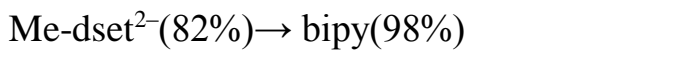 \\
\hline & & & & $112 \rightarrow 118$ & 39 & $\operatorname{bipy}(85 \%) \rightarrow \operatorname{bipy}(93 \%)$ \\
\hline \multirow[t]{3}{*}{ S23 } & 4.357 & 284.5 & 0.062 & $109 \rightarrow 118$ & 32 & $\operatorname{Pt}(42 \%)+$ Me-dset ${ }^{2-}(31 \%) \rightarrow \operatorname{bipy}(93 \%)$ \\
\hline & & & & $114 \rightarrow 120$ & 22 & Me-dset $^{2-}(82 \%) \rightarrow \operatorname{bipy}(98 \%)$ \\
\hline & & & & $115 \rightarrow 121$ & 18 & Me-dset $^{2-}(99 \%) \rightarrow$ Me-dset $^{2-}(42 \%)+\operatorname{Pt}(39 \%)$ \\
\hline S24 & 4.363 & 284.1 & 0.066 & $115 \rightarrow 121$ & 69 & $\operatorname{Me}^{-\operatorname{dset}^{2-}(99 \%) \rightarrow \text { Me-dset }^{2-}(42 \%)+\operatorname{Pt}(39 \%)}$ \\
\hline S27 & 4.434 & 279.6 & 0.069 & $110 \rightarrow 118$ & 80 & $\operatorname{Pt}(53 \%)+$ Me-dset ${ }^{2-}(34 \%) \rightarrow \operatorname{bipy}(93 \%)$ \\
\hline \multirow[t]{2}{*}{ S29 } & 4.478 & 276.9 & 0.373 & $109 \rightarrow 118$ & 44 & $\operatorname{Pt}(42 \%)+\operatorname{Me}^{-d_{s e t}{ }^{2-}(31 \%) \rightarrow \operatorname{bipy}(93 \%)}$ \\
\hline & & & & $112 \rightarrow 118$ & 31 & $\operatorname{bipy}(85 \%) \rightarrow \operatorname{bipy}(93 \%)$ \\
\hline S30 & 4.596 & 269.8 & 0.016 & $115 \rightarrow 122$ & 92 & Me-dset ${ }^{2-}(99 \%) \rightarrow$ Me-dset $^{2-}(99 \%)$ \\
\hline
\end{tabular}

\footnotetext{
${ }^{\text {a }}$ The MOs are labeled according to Figure 3.
} 
Table S11. Static first hyperpolarizabilities $\beta_{t o t}$ (a.u. and esu) and static dipole moments $\mu$ (D) calculated for 1 and $\mathbf{2}$ in the gas phase and in $\mathrm{CH}_{2} \mathrm{Cl}_{2}$ (IEF-PCM SCRF model).

\begin{tabular}{clcc}
\hline & & $\mathbf{1}$ & $\mathbf{2}$ \\
\hline \multirow{3}{*}{$\mathrm{Gas}$} & $\beta_{\mathrm{tot} \cdot 10^{4} \text { (a.u.) }}$ & 4.26 & 4.81 \\
& $\beta_{\mathrm{tot} \cdot 10^{-30}(\mathrm{esu})}$ & 368 & 416 \\
& $|\mu|(\mathrm{D})$ & 7.45 & 7.95 \\
& $\beta_{\mathrm{tot} \cdot 10^{4} \text { (a.u.) }}$ & 3.97 & 4.06 \\
$\mathrm{CH}_{2} \mathrm{Cl}_{2}$ & $\beta_{\mathrm{tot} \cdot 10^{-30}(\mathrm{esu})}$ & 343 & 351 \\
& $|\mu|(\mathrm{D})$ & 11.08 & 11.66 \\
\hline
\end{tabular}




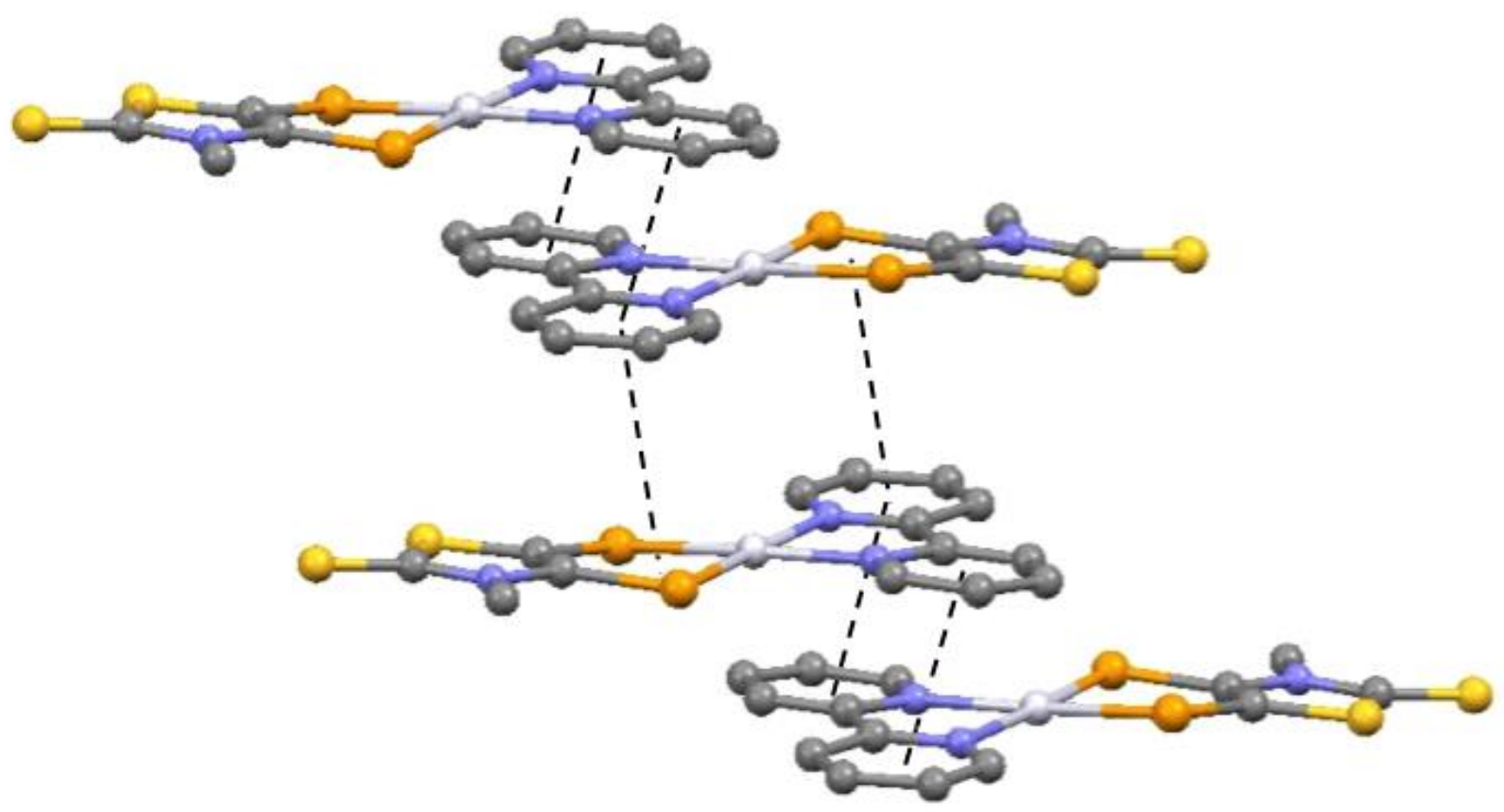

Figure S1. Drawing of a portion of the crystal packing of $1 \cdot 1 / 2 \mathrm{CH}_{2} \mathrm{Cl}_{2}$. Hydrogen atoms were omitted for clarity. 


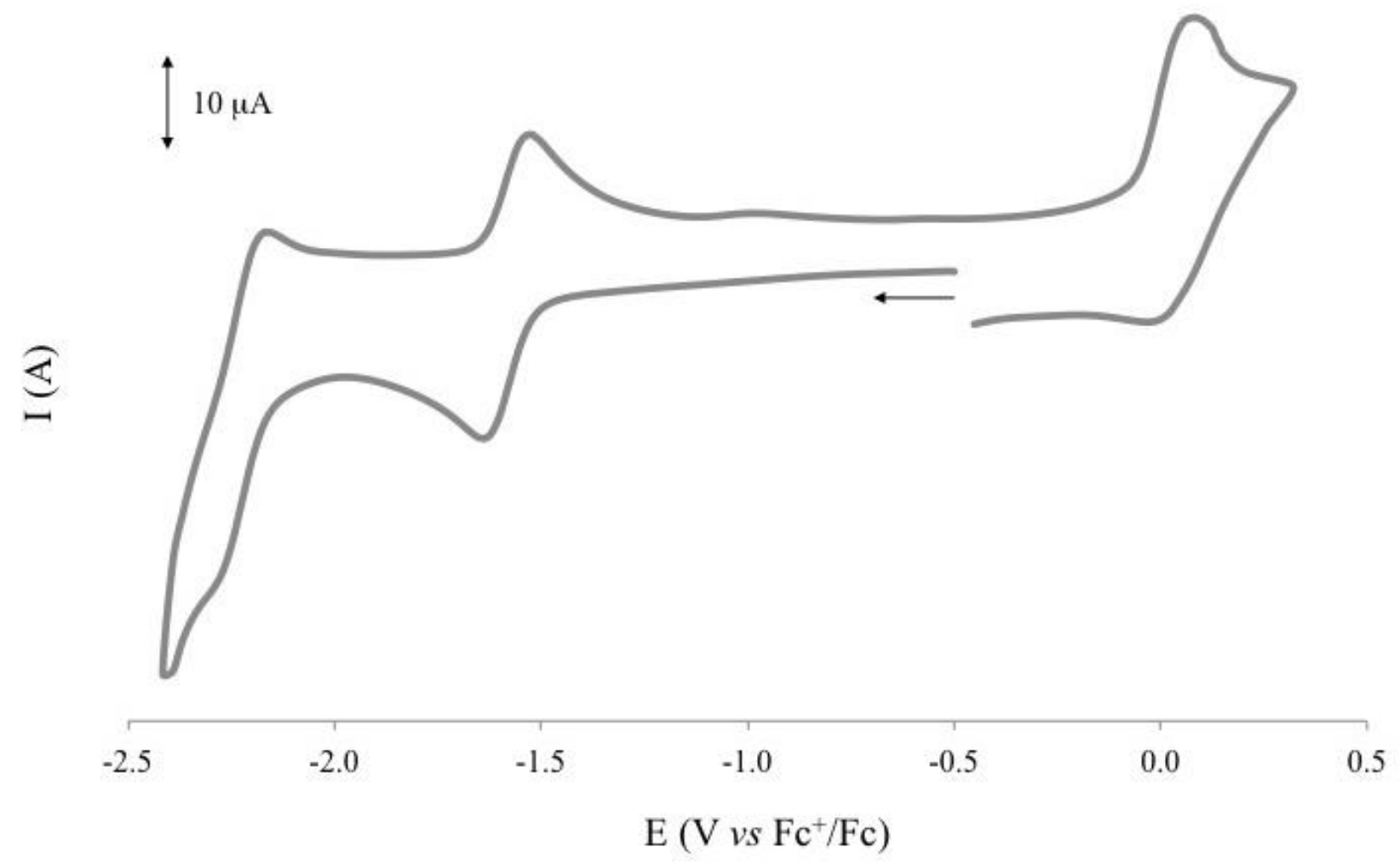

Figure S2. Cyclic voltammogram recorded at a platinum electrode on an anhydrous dmso solution of 1 [298 K; scan rate $50 \mathrm{mV} \mathrm{s}^{-1}$; supporting electrolyte $\left.\left(\mathrm{TBA}^{+}\right)\left(\mathrm{PF}_{6}^{-}\right) 0.1 \mathrm{M}\right]$. 


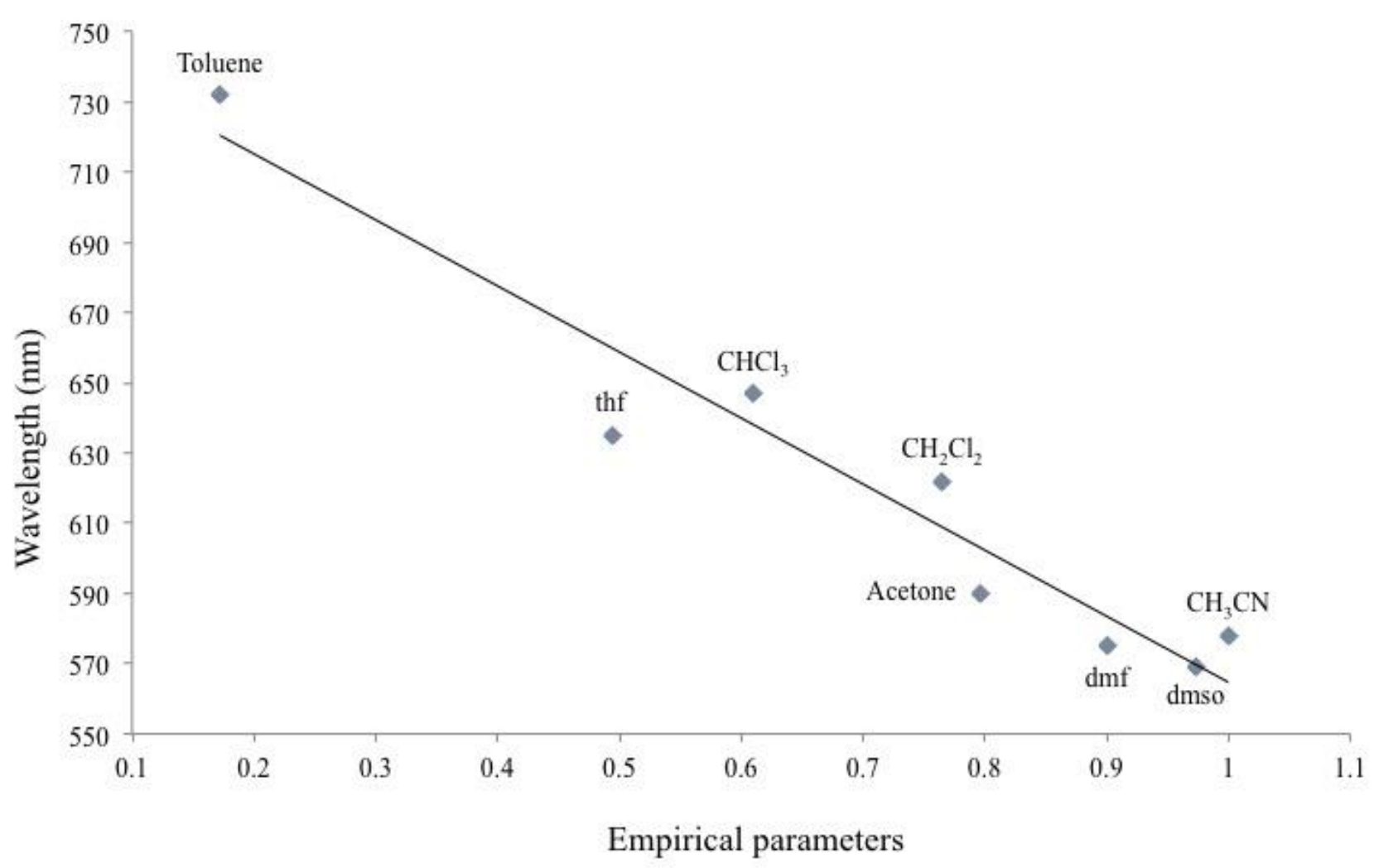

Figure S3. Correlation between the absorption maxima $\lambda_{\max }$ of the solvatochromic absorption in different solvents for complex $\mathbf{1}$ and the empirical scale formulated by Eisenberg for $\left[\operatorname{Pt}\left(\mathrm{N}^{\wedge} \mathrm{N}\right)\left(\mathrm{S}^{\wedge} \mathrm{S}\right)\right]$ complexes $\left(R^{2}=0.93\right.$; Table 1 ; ref. 24$)$. 


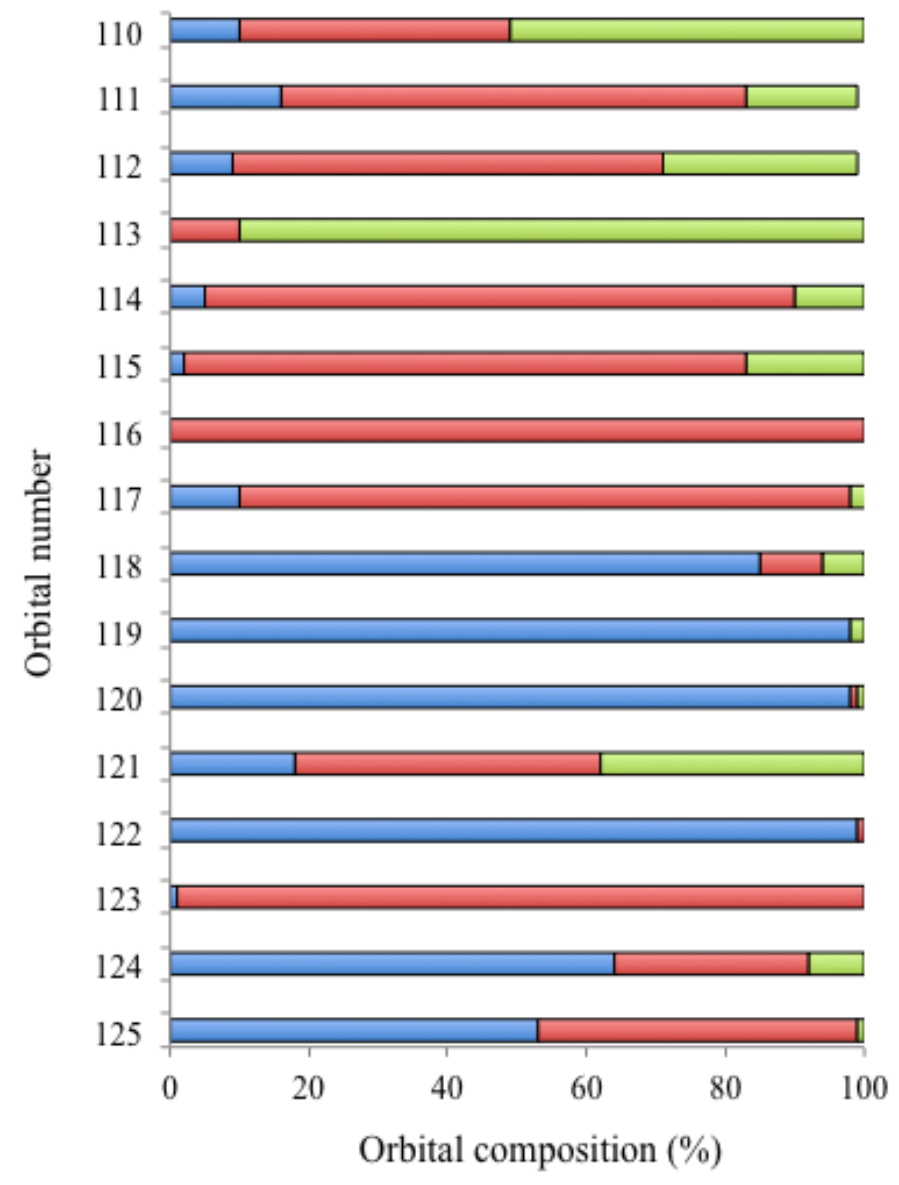

Figure S4. Frontier molecular orbital (KS-MOs 110-125; HOMO $=117$, LUMO $=118$ ) composition calculated for $\mathbf{1}$ in the gas phase [fragments: platinum atom (green); Me-dset ${ }^{2-}$ ligand (red); 2,2'-bipyridine (blue)]. 


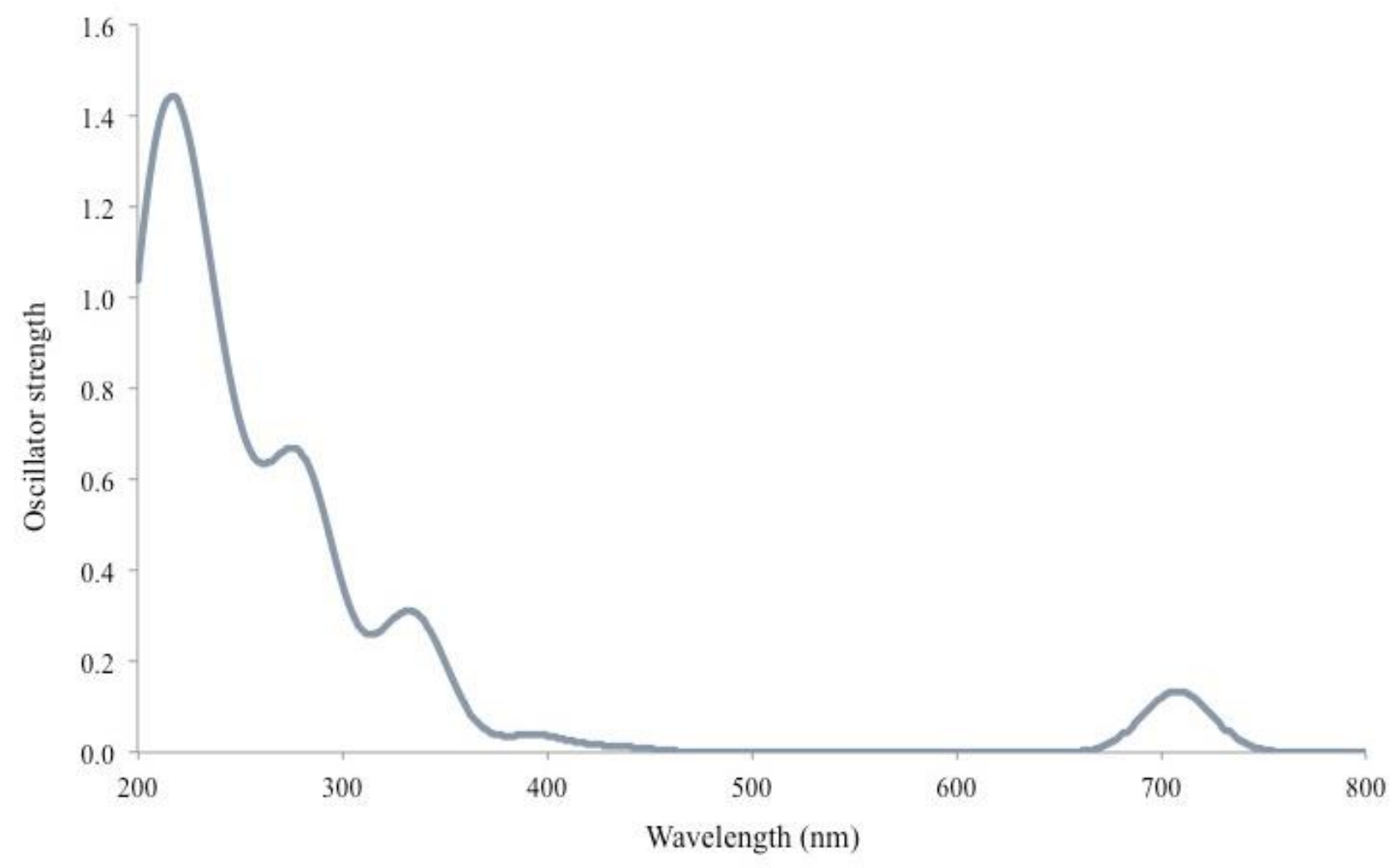

Figure S5. TD-DFT simulated UV-Vis absorption spectrum (200-800 nm) of complex $\mathbf{1}$ in $\mathrm{CH}_{2} \mathrm{Cl}_{2}$ (IEF-PCM SCRF model; Table S7). 


\section{References for Supporting Information}

1 G. T. Morgan, F. H. Burstall, J. Chem. Soc., 1934, 965-971.

2 J. V. Rund, Inorg. Chem., 1974, 13, 738-740.

3 S. Eid, M. Formigué, T. Roisnel, D. Lorcy, Inorg. Chem., 2007, 46, 10647-10654.

4 A. Pintus, M. C. Aragoni, N. Bellec, F. A. Devillanova, D. Lorcy, F. Isaia, V. Lippolis, R. A. M. Randall, T. Roisnel, A. M. Z. Slawin, J. D. Woollins, M. Arca, Eur. J. Inorg. Chem., 2012, 3577-3594.

5 http://www.unipress.waw.pl/fityk/.

6 G. M. Sheldricks, SHELXL-97, University of Göttingen, Germany, 1997.

7 G. M. Sheldricks, SHELXS-97, University of Göttingen, Germany, 1997.

Sheldrick, G. M. (2008). Acta Cryst. A64, 112-122.

8 W. Koch, M. C. Holthausen, “A Chemist's Guide to Density Functional Theory”, 2nd ed., Wiley-VCH, Weinheim, Germany, 2002.

9 Gaussian 09, Revision A.02, M. J. Frisch, G. W. Trucks, H. B. Schlegel, G. E. Scuseria, M. A. Robb, J. R. Cheeseman, G. Scalmani, V. Barone, B. Mennucci, G. A. Petersson, H. Nakatsuji, M. Caricato, X. Li, H. P. Hratchian, A. F. Izmaylov, J. Bloino, G. Zheng, J. L. Sonnenberg, M. Hada, M. Ehara, K. Toyota, R. Fukuda, J. Hasegawa, M. Ishida, T. Nakajima, Y. Honda, O. Kitao, H. Nakai, T. Vreven, J. A. Montgomery Jr., J. E. Peralta, F. Ogliaro, M. Bearpark, J. J. Heyd, E. Brothers, K. N. Kudin, V. N. Staroverov, R. Kobayashi, J. Normand, K. Raghavachari, A. Rendell, J. C. Burant, S. S. Iyengar, J. Tomasi, M. Cossi, N. Rega, J. M. Millam, M. Klene, J. E. Knox, J. B. Cross, V. Bakken, C. Adamo, J. Jaramillo, R. Gomperts, R. E. Stratmann, O. Yazyev, A. J. Austin, R. Cammi, C. Pomelli, J. W. Ochterski, R. L. Martin, K. Morokuma, V. G. Zakrzewski, G. A. Voth, P. Salvador, J. J. Dannenberg, S. Dapprich, A. D. Daniels, O. Farkas, J. B. Foresman, J. V. Ortiz, J. Cioslowski, D. J. Fox, Gaussian, Inc., Wallingford CT, 2009.

10 C. Adamo, V. Barone, J. Chem. Phys., 1999, 110, 6158-6170.

11 A. Schäfer, H. Horn, R. Ahlrichs, J. Chem. Phys., 1992, 97, 2571-2577.

12 W. C. Ermler, R. B. Ross, P. A. Christiansen, Int. J. Quant. Chem., 1991, 40, 829-846.

13 T. H. Dunning Jr., P. J. Hay in "Methods of Electronic Structure, Theory”, Vol. 2, H. F. Schaefer III ed., Plenum Press, 1977.

14 J. V. Ortiz, P. J. Hay, R. L. Martin, J. Am. Chem. Soc., 1992, 114, 2736-2737.

15 Average calculated Pt-Se bond length $=2.386$ and $2.403 \AA$ for the calculations performed with Ahlrichs pVDZ and CRENBL+ECP, respectively.

16 A. E. Reed, R. B. Weinstock, F. Weinhold, J. Chem. Phys., 1985, 83, 735-746.

17 K. Wiberg, Tetrahedron, 1968, 24, 1083-1096.

18 J. Tomasi, B. Mennucci, R. Cammi, Chem. Rev., 2005, 105, 2999-3094.

19 M. P. Cifuentes, M. G. Humphrey, J. Organomet. Chem., 2004, 3968-3981.

20 P. J. Mendes, A. J. P. Carvalho, J. P. P. Ramalho, J. Mol. Struct.: THEOCHEM, 2009, 900, 110-117.

21 Æ. Frisch, H. P. Hratchian, R. D. Dennington II, T. A. Keith, J. Millam, A. B. Nielsen, A. J. Holder, J. Hiscocks. Gaussian, Inc. Gauss View Version 5.0, 2009. 
22 G. Schaftenaar, J. H. Noordik, J. Comput.-Aided Mol. Des., 2000, 14, 123-134.

23 N. M. O’Boyle, A. L. Tenderholt, K. M. Langner, J. Comput. Chem., 2008, 29, 839-845.

24 S. D. Cummings, R. Eisenberg, J. Am. Chem. Soc., 1996, 118, 1949-1960. 